\title{
Simultaneous Analysis of Vibrations and Noise in the Task of Minimizing Vibroacoustic Activity of Machines
}

\author{
Zbigniew DĄBROWSKI, Jacek DZIURDŹ \\ Institute of Machine Design Fundamentals, Warsaw University of Technology \\ Narbutta 84, 02-524 Warszawa, Poland; e-mail: zdabrow@simr.pw.edu.pl
}

(received March 10, 2016; accepted April 7, 2016)

\begin{abstract}
Simultaneous propagation of vibrations and noise has an important role in the task of minimizing vibroacoustic hazards on the station of operator of the construction machinery. In many cases vibrations transferred by the construction are processed to noise in different points of the machine. As a result, they may increase the level of noise at the workplace. The paper presents the proposition of a simple estimation of noise and vibration propagation paths of the machine. On the basis of the analysis of hydraulic excavator an effectiveness of a proposed procedure was shown. This procedure helps to minimize the transfer of vibrations of power unit in selected frequency ranges which led to the change of overall noise level in operator's cab about $5 \mathrm{~dB}$.
\end{abstract}

Keywords: signal separation; noise minimizing; frequency response function.

\section{Introduction}

Modern manufactured machines are optimized due to the three basic criteria which result from economic assumptions. These are the following criteria: minimizing the use of energy and minimizing weight and dimensions. As a result, there are obtained constructions with relatively low stiffness and very often with a highly complex structure. Such constructions are susceptible to dynamic deformations, which naturally caused a rapid development of modelling methods, possible due to the development of digital simulation techniques. However, the construction of the "exact" structural model, , which allows to describe the ways of propagation of vibroacoustic energy at the design stage, is still impossible. For the purposes of technical applications there is still a problem of acceptance of model simplifications (degree of model abstraction) and its identification (BATKO et al., 2008).

In the latter case, the measuring capabilities and assumed assessment techniques play an important role. These techniques result from applied methods of signal analysis, including inevitable errors which are strictly connected with these techniques. It can be concluded that there still exist two contradicting trends. The first one uses FEM and BEM techniques and it aims at creating more detailed models (MADEJ, 2003). The second one improves techniques which bring out more de- tailed information by observing the relations between input and output of the model, and the identification of frequency response function (DĄBROWSKI, 1992; DĄBROWSKi et al., 2013; DziURdź, 2013a; 2013b).

In both cases there are serious difficulties in the analysis (description and observation) of the change of vibration energy into noise and vice versa. It leads to independently solved tasks of minimization of vibrations and noise, which affect the operator of the machine. The authors claim that in many cases it causes serious mistakes. Moreover, it increases the number of searches of the constructional solutions of sound- and vibroisolating structures in experimental way which sometimes is very expensive. Meanwhile, the development of digital measurement techniques allows, on certain level of abstraction, for identification of ways of propagation of vibroacoustic energy, including mutual transformations.

This study analyses the use of filter features of coherence function and frequency response function calculated with the use of cross-spectrum (BENDAT, Piersol, 1993; 2010; Dziurdź, 2013a).

\section{Theoretical considerations}

Let's treat the machine as a set of independent sources of noise and mechanical vibrations, where some 
of them are correlated with each other and some of them are not. Initially it can be also assumed that we know their characteristics (from a model description or observation) and we observe the effects of their influence in a selected point (output) (Fig. 1). It can be, e.g. the position of the machine operator, the selected point of external field, or the point where we observe vibroacoustic process for diagnostic purposes.

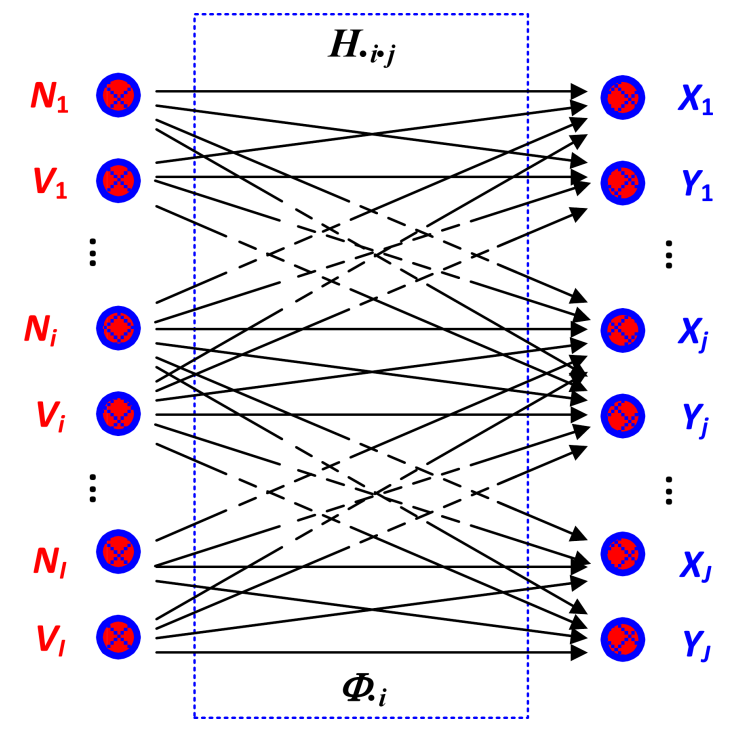

Fig. 1. Block diagram of the machine.

The relation input $\leftrightarrow$ output in frequency domain is written as follows:

$$
\begin{aligned}
& \underset{\omega}{\mathcal{S}} \mathcal{F} \underset{t}{\mathcal{S}}\left\{x_{i}(t \ldots)\right\}=X_{i}=\sum_{j=1}^{J} V_{j} \cdot H_{V j X i} \\
& +\sum_{j=1}^{J} N_{j} \cdot H_{N j X i}+\Phi_{X i}+\Psi \\
& \underset{\omega}{\mathcal{S}} \mathcal{F} \underset{t}{\mathcal{S}}\left\{y_{i}(t \ldots)\right\}=Y_{i}=\sum_{j=1}^{J} V_{j} \cdot H_{V j Y i} \\
& +\sum_{j=1}^{J} N_{j} \cdot H_{N j Y i}+\Phi_{Y i}+\Psi
\end{aligned}
$$

where $\left\{x_{i}(t \ldots)\right\}$ and $\left\{y_{i}(t \ldots)\right\}$ - observed output of the system - vibration or noise at the selected point. The effect of the measurement is a random process dependent on time and other defined variables as needed, such as operation time of the machine, coordinate of the fixing point of sensor, or the indicator of tested specimen; $\mathcal{S}_{t}$ and $\underset{\omega}{\mathcal{S}}$ are respectively the operators of selection and averaging in time and frequency domain. The introduction of these operators is necessary due to the possibility of comparing the results of measurements with a determined model. It is also purposeful due to the choice of signal parameters, which we intend to analyse; $\mathcal{F}$ - Fourier transform; $V$ and $N$ represent respectively the Fourier spectrum of sources of vibrations and noise. In technical applications it is important to scale these quantities in real energy units or appropriate normalization when we perform dimensionless analysis.

$H_{V i X j}, H_{V i Y j}, H_{N i X j}$ and $H_{N i Y j}$ are the frequency response function of vibrations and noise on the path source $\leftrightarrow$ observation point. When it is possible to extract subsystems of elements with frequency response functions known from a model description or from the research, then total frequency response function is usually written in the form of product:

$$
H=\bigcap_{i=1}^{I} H_{i} .
$$

It is worth noting that such a way of describing the identification of individual components allowed the authors of this article to develop an algorithm of selection of parameters of sound-isolating screens of drive system of the machine (DAzBRowsKi et al., 2013).

$\Phi$ is a function describing the error of the assumed model, including the error of linear approximation of propagation model adopted, including linear approximations of propagation paths; $\Psi$ is an inevitable error of measurement.

Complete identification of such a model requires a description of all paths of propagation, including the points, where processing of mechanical vibrations into noise and vice versa, takes place. It would require a vast number of measurements. It should be noticed that even when the number of points of observation equals (or is bigger) the number of sources, the number of unknown functions will equal at least $2 n+1$, taking for $n$ the number of sources and including only total function $\Phi$. It should be noticed that defining the "real source of noise" inside the machine structure is usually impossible. Typically, in accordance with the methodology specified, for example, in the paper (DAzBROWSKI et al., 2013), there are made series of measurements on the basis of the measurements in direct neighbourhood of the supposed source. What is more, the source is described with such a model (material point or system of material points) in order to obtain a high compliance in these measuring points. This statement is significant because frequency response functions defined in the process of identification do not have to be compatible with the frequency response function defining exactly the physical energy flow between the extortion and dynamic response of the system. These functions constitute the characteristics of a two-dimensional random process whose components are mechanical vibrations or noise in one selected point of the machine, which is assumed as a source and a similar vibracoustic process treated as output.

Complete identification of a model described with Eqs. (1) needs the solution of the system containing over two times bigger number of unknowns than the 
number of equations. That is why, it is important to find (define) additional dependencies. Assuming that in order to make the proceedings more readable, we will omit "indirect" transformations of vibration energy into noise or vibrations in another output point, and we will limit the considerations to total frequency response functions, we will obtain:

$$
\begin{aligned}
& X_{i}=\sum_{j=1}^{J} V_{j} \cdot H_{V j X i}+\sum_{j=1}^{J} N_{j} \cdot H_{N j X i}+\Phi_{i}+\Psi, \\
& Y_{i}=\sum_{j=1}^{J} V_{j} \cdot H_{V j Y i}+\sum_{j=1}^{J} N_{j} \cdot H_{N j Y i}+\Phi_{i}+\Psi, \\
& f_{i}\left(H_{V j X i}, \ldots H_{N j X i}, \ldots H_{V j Y i}, \ldots H_{N j Y i}, \ldots\right)=0, \\
& \rho \stackrel{\text { def }}{=} \rho(\ldots, \ldots),
\end{aligned}
$$

This system of equations is solvable for $\Phi_{i}+\Psi=0$, with an assumed error $\delta=\Phi_{i}+\Psi$ providing that the metric is defined properly. When we want to find functions $\Phi_{i}$ we need $i$ of additional relations. Dependencies $\Phi_{i}$ may be defined in a model (analytical) way, accepting inevitable error of modelling or defining them empirically as relations between $N_{i}$ and $V_{i}, X_{j}$ and $H_{V i X j}$, which constitutes the proposition of authors. We will follow the procedure on the following example.

\section{The example of noise minimization in a cabin of excavator operator}

Hydraulic excavator was the object of the research. Drive system containing combustion engine was the main source of noise and vibrations for the excavator. Vibroacoustic structure of such a machine is typical for a wide class of working machines. There are numerous mechanisms and supporting structures between the power unit and the station of operator which usually is the main sphere of minimization of the influence of vibroacoustic hazards. The propagation path related to these mechanisms and structures is very difficult to model. Various "engineering" guidelines of improving the acoustic and vibration comfort in the operator's cab usually come down to fixing flat sound-isolating covers around the engine (drive system) and increasing the acoustic insulation of the walls of operator's cage. It should not be forgotten that the complexity of propagation paths of vibroacoustic energy between the source and output very often causes the following effects:

1. The spectral structures of noise inside the housing of power unit and inside the operator's cab may be significantly different in frequency scale, which confirms the nonlinear processing on the propagation path.

2. There exist the points where the vibration energy is processed into noise (and vice versa), which with the external measurement of acoustic field results in external noise sources.

3. The introduction of additional flat screens inside the complex structure in the absence of free choice of distance can cause standing wave effect (volume resonance) or such a strong boost inside the sound-isolating housing that other paths of propagation will activate.

For these reasons, the relations between noise propagation and mechanical vibrations are discussed in the analysed example.

The block diagram of the machine is shown in Fig. 2. The observed noise inside the housing of drive system is assumed as the "source" of noise. Of course, this is not the base source in the physical sense. The combustion processes in the engine, the noise of gear, hydraulic pump and sounds of auxiliary engine mechanisms are such sources. Engine enclosure causes such a composition of processes coming from these sources that, in practice, there is acoustic field in all its points, with equal or almost equal level. Mechanical vibrations transferred to construction (frame) were assumed as the "source" of vibrations behind vibroisolating elements of power unit. Such assumptions allow to assume a simplified model with two inputs and two outputs shown in Fig. 3.

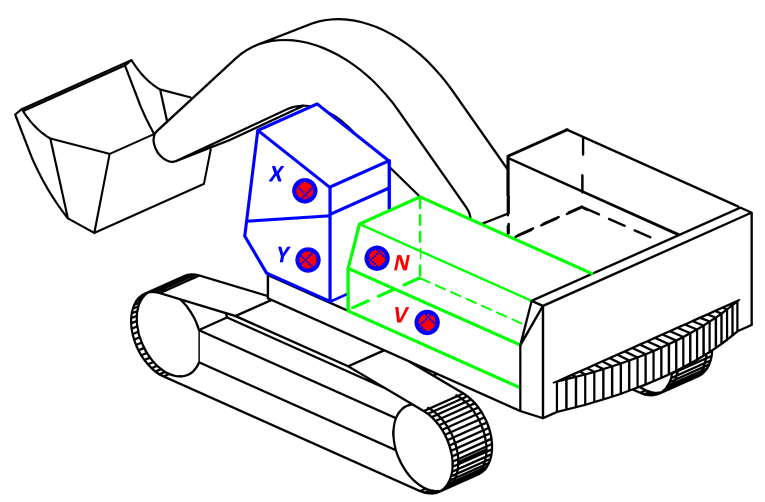

Fig. 2. Hydraulic excavator with a marked measurement points.

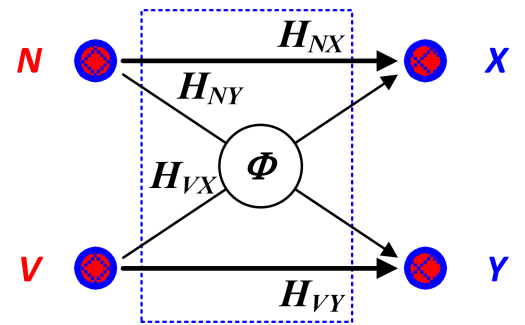

Fig. 3. Simplified model of noise and vibration propagation in hydraulic excavator.

The results of the observation of inputs and outputs, i.e. vibration and noise in the engine compartment and the operator's cage are shown in Fig. 4. 
a)

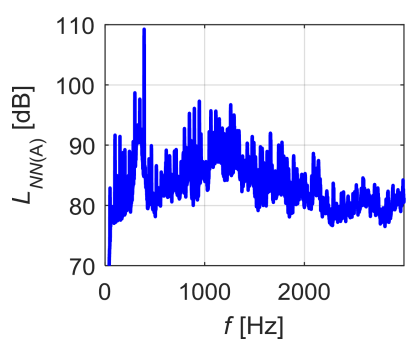

c)

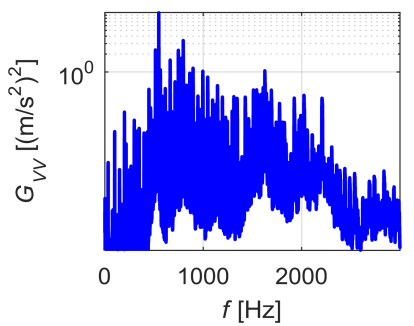

b)

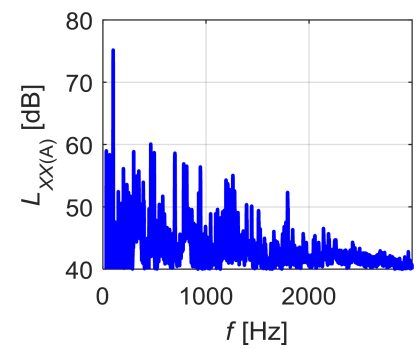

d)

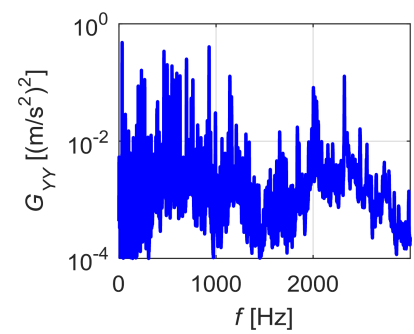

Fig. 4. The spectral power density: a) noise in the engine compartment $\left.L_{N N(\mathrm{~A})}, \mathrm{b}\right)$ noise in the operator's cab $L_{X X(\mathrm{~A})}$, c) vibration accelerations of engine suspension $G_{V V}$, d) vi-

bration accelerations of operator's cab floor $G_{Y Y}$.

Model assumed in accordance with dependencies (1) describes the system of equations:

$$
\begin{aligned}
& X=V \cdot H_{V X}+N \cdot H_{N X}+\Phi_{x}, \\
& Y=V \cdot H_{V Y}+N \cdot H_{N V}+\Phi_{y} .
\end{aligned}
$$

As it can be seen, this system of two equations has six unknowns. Assuming that functions $\Phi_{x}$ and $\Phi_{y}$ are "small" and allowing for some errors, it is possible to reduce the number of unknowns to four and the system still remains unsolvable. Therefore, it is necessary to find "extra" relations between frequency response functions $H$. First, there were calculated the functions of coherence with the following formula:

$$
\gamma_{A B}^{2}=\frac{\left|G_{A B}\right|^{2}}{G_{A A} \cdot G_{B B}},
$$

where $G_{A B}$ cross-spectral density (A and B) and $G_{A A}$ and $G_{B B}$ spectral density of inputs and outputs, respectively.

As it is known, function $\gamma^{2}$ equal to 1 for no disturbance and linear relation input $\leftrightarrow$ output. Figure 5 shows the functions of coherence between the noise in the operator's cab and vibrations, and noise sources. Even "rough" analysis shows that vibrations "sources" are more coherent with the noise of operator's cab than the noise in the input. It leads to defining the participation of mechanical vibrations in the spectrum of output noise. Low values $\gamma_{N X}^{2}$ in many bands allow for

a)

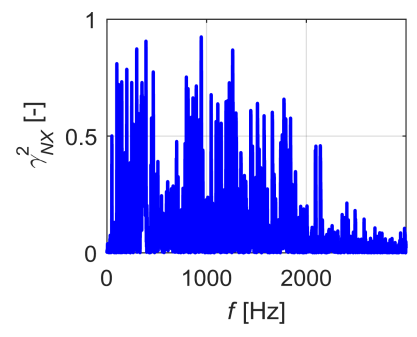

b)

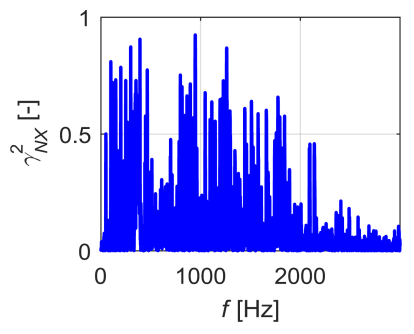

c)

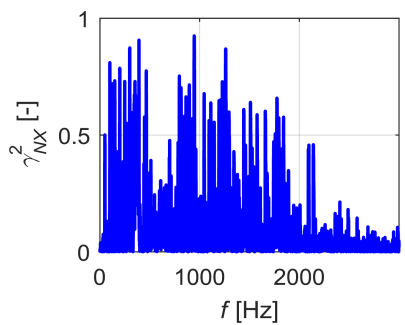

Fig. 5. Coherence functions of: a) noise in engine compartment and noise in operator's cab $\gamma_{N X}^{2}$, b) vibration accelerations of engine suspension and noise in operator's cab $\gamma_{V X}^{2}$, c) vibration accelerations of engine suspension and vibration accelerations of operator's cab $\gamma_{V Y}^{2}$.

the assumption of a weak correlation of the processes that make up the noise in the cabin and sources caused by the noise and vibrations. Let's mark the noise component in the cabin which comes from mechanical vibrations and other unknown sources with $Z$ according to the formula:

$$
V \cdot H_{V X}+\Phi=Z
$$

Next, we should calculate the frequency response function $H_{N X}$ in two ways, using a cross-spectral density of the processes $N$ and $X$ treating the process $Z$ as a disturbance in the system output (Fig. 6). It results from the filter properties of frequency response function (Bendat, Piersol, 1993; RAndall, 1987) that:

$$
\begin{aligned}
& H_{N X}^{(1)}=\frac{G_{N Z}}{G_{N N}}=\frac{G_{N X}}{G_{N N}}=H_{N X}(\mathrm{i} \omega) \\
& H_{N X}^{(2)}=\frac{G_{X X}}{G_{X N}}=H_{N X}\left(1+\frac{G_{Z Z}}{G_{X X}-G_{Z Z}}\right)
\end{aligned}
$$

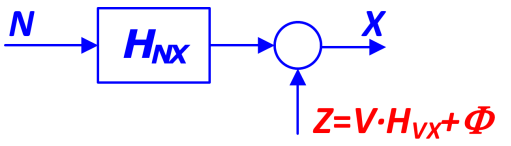

Fig. 6. Relationship between noise and mechanical vibrations in operator's cab. 
using the fact that:

$$
\gamma_{N X}^{2}=\frac{H_{N X}^{(1)}}{H_{N X}^{(2)}}
$$

leads to the dependency:

$$
\left(1-\gamma_{N X}^{2}\right) G_{X X}=G_{Z Z}
$$

allowing for easy determination of disturbance of spectral density $G_{Z Z}$ thereby the model of frequency response function $\left|H_{N X}\right|$. Determination of phase response and complete frequency response function is also possible, however, it is not necessary in the proposed algorithm. Figure 7 presents the part of noise which comes from uncorrelated part of mechanical vibrations propagated by construction in the spectrum of total noise on operator's station, limited to dominant components $\mathcal{S} G_{Z Z}$ (in this example 30 components). From theoretical point of view, it is the way to find one of the missing equations which are necessary to describe propagation paths. Function $H_{V Y}$ can be calculated analogically. It is also possible to define functions $\Phi$ analogically by studying the relations between $N, V$ and $X, Y$.

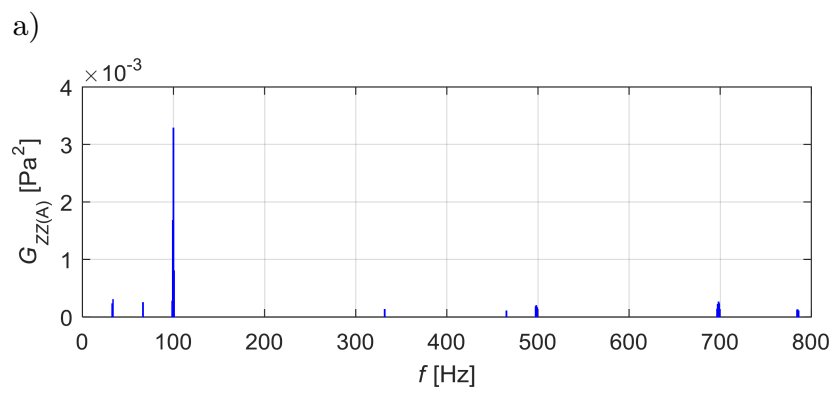

b)

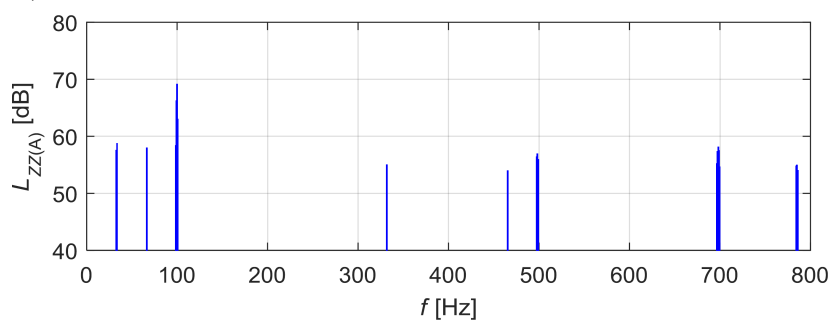

Fig. 7. Determined separated values of "disturbance": a) spectral density of power $G_{Z Z}$, b) spectral density power recalculated to values of noise levels $L_{Z Z(\mathrm{~A})}$ for $\underset{\omega}{\mathcal{S}}=\underset{\omega 30}{\mathcal{S}}$.

For practical reasons the selected part of mechanical vibrations in total noise allowed for optimization of the engine support in order to eliminate the vibrations in dominant bands. The effect of influence of this minimization on the noise in operator's cab is shown in Fig. 8.

It is worth noting that the practical elimination of vibration propagation path caused a slightly larger (approx. $5 \mathrm{~dB}$ ) decrease in the noise level in the cab, a)

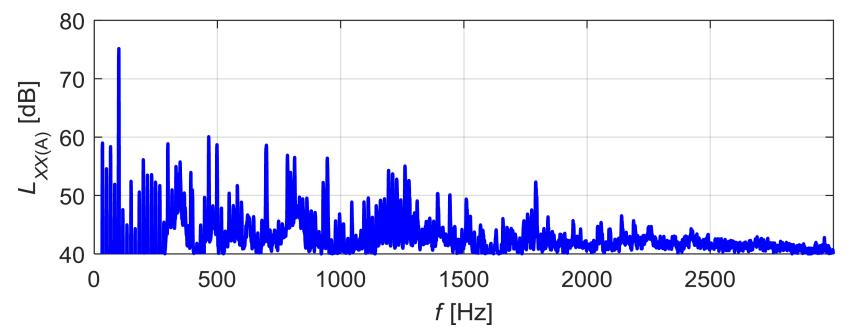

b)

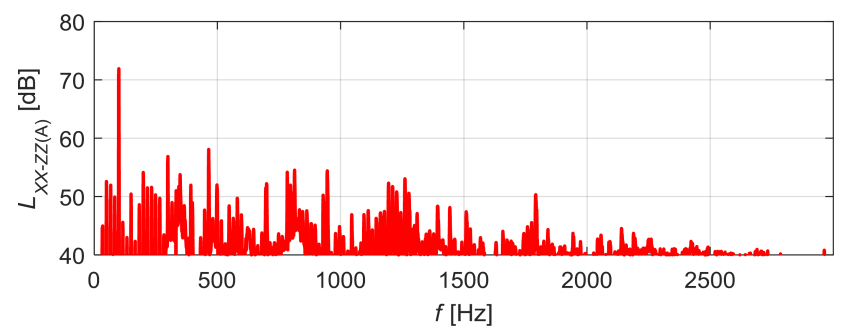

Fig. 8. The effect of minimizing the vibration impact on the noise in operator's cab: spectral power density of noise in operator's cab $L_{X X(\mathrm{~A})}$ and spectral power density of noise in operator's cab $L_{X X-Z Z(\mathrm{~A})}$ after taking into account the influence of "disturbance" $G_{Z Z}$.

which results from the elimination of correlated vibrations which are not taken into account by the proposed procedure.

\section{Conclusion}

The example confirms the usefulness of methodology proposed by the authors. This methodology concerns the approach to the task of minimizing disturbances with the noise of operators of modern (light and elastic) construction machinery, where the power unit containing diesel engine is the main source of vibroacoustic processes. Despite the fact that an independent description of the main propagation paths of noise often allows for a selection of optimal characteristics of sound-isolating structures, total analysis of vibrations and noise allows for a higher efficiency of engineering activities. It should be used even when mechanical vibrations do not exceed acceptable level. Extracting correlated and not correlated (non-coherent) elements of observed signals and properly defined "outputs" and "inputs" of the system are the basis for correctness and effectiveness of the analyses. A simple example of a model with two inputs shows that the way of describing and searching for additional dependencies between frequency response functions can be easily generalized into a model with many inputs and outputs. The proposed technique of separating a part of signal which comes from a different source (in this case from mechanical vibrations) may be also used for extracting the information part of signal for technical diagnostic needs. 


\section{References}

1. Batko W., Dabrowski Z., Kicinski J. (2008), Nonlinear Effects in Technical Diagnostics, Publishing and Printing House of the Institute for Sustainable Technologies - NRI.

2. Bendat J.S., Piersol A.G. (1993), Engineering Applications of Correlation and Spectral Analysis, 2nd ed., John Wiley, New York.

3. Bendat J.S., Piersol A.G. (2010), Random data: Analysis and Measurement Procedures, 4th ed., John Wiley, New York.

4. Crocker M.J. [Ed.], (2007), Handbook of Noise and Vibration Control, John Wiley \& Sons.

5. Dabrowski Z. (1992), The Evaluation of the Vibroacoustic Activity for the Needs of constructing and use of Machines, Machine Dynamics Problems, Vol. 4, Warsaw.

6. DĄbrowski Z., Dziurdź J., PAkowski R. (2013), Selection of Sound Insulating Elements in Hydraulic Excavators Based on identification of Vibroacoustic Energy Propagation Paths, Archives of Acoustics, 38, 4, 471-478.
7. DziURdz J. (2000), Minimize noise and vibration for the operator working machine (proposed method), [in Polish: Minimalizacja hałasu i drgań na stanowisku operatora maszyny roboczej (propozycja metody)], $\mathrm{PhD}$ Dissertation, Warsaw University of Technology.

8. Dziurdz J. (2013a), Application of Correlation and Coherence Functions in Diagnostic Systems, Selected, peer reviewed papers from the Symposium on Mechatronic Systems, Mechanics and Materials, Solid State Phenomena, 196, 3-12.

9. Dziurdź J. (2013b), Analysis of nonlinear phenomena in diagnosing of the vehicle drive systems, [in Polish: Analiza zjawisk nieliniowych $w$ diagnozowaniu uktadów napędowych pojazdów], Publishing and Printing House of the Institute for Sustainable Technologies - NRI.

10. Madej H. (2003), Minimisation of Vibro-Acoustic Activity in Toothed Gears, [in Polish: Minimalizacja aktywności wibroakustycznej korpusów przektadni zębatych], Publishing and Printing House of the Institute for Sustainable Technologies - NRI.

11. Randall R.B. (1987), Frequency Analysis, Brüel \& Kjær. 